\title{
Implementation of a System for Physiological Status Monitoring by using Tactical Military Networks
}

\author{
Goce Stevanoski \#,*, Ivica Kocev!, Jugoslav Achkoski”, Saso Koceski!, and Boban Temelkovski ${ }^{\#}$ \\ "Military Academy, General Mihailo Apostolski - Skopje, Macedonia \\ 'Faculty of Computer Science, Stip, Macedonia \\ ${ }^{\#}$ E-mail: gstevanos@gmail.com
}

\begin{abstract}
E-health sensors are continuing to become more advanced and more reliable in monitoring the human physiological status. There is a continuous scope for improvement in their implementation in different emergency situations. Military organisations can take an advantage of this technology for applying physiological status monitoring on personnel engaged in military operations. This implementation is driven by continuous enhancements of existing communication equipment that produces more data capable radio networks in military environment. Based on these technologies we are proposing system communication architecture for applying real-time physiological status monitoring for personnel engaged in military operations. To examine the proposed architecture, a laboratory testing was performed. The laboratory work included a definition of military communication equipment, testing the received data with custom developed algorithm based on Markov decision process for automating the medical emergency protocol (MDP-AMEP) and implementation of adequate data protocols for data transmitting. Obtained results showed that physiological status of the military personnel can be successfully monitored by using tactical military network.
\end{abstract}

Keywords: E-health sensors, UHF radio communication, Markov decision process, first responder, physiological status monitoring

\section{INTRODUCTION}

Continuous advancement in IT industry has provided the possibility to use this technology in many different emergency situations. Military organisations can take an advantage of this new technology to set up a system for monitoring the physiological status of personnel engaged in military operations.

FitzGerald ${ }^{1}$, et al., provided comprehensive review of the evolution of the triage process in the hospitals' emergency departments. While various triage protocols, described in their study, differ in their methodology, all of them classify the patients in terms of urgency.

Ellebrecht \& Kaufmann ${ }^{2}$ clearly verify the impact of information technologies (IT) on efficient rescue operation, particularly by reducing the time to allocate casualties to hospitals. The authors prove that graphical representation could be very beneficial at it can reduce the uncertainties.

Horne $^{3}$, et al. have evaluated the performances of UK Triage Sieve algorithm in practice and compared it with the military Triage Sieve version (MS). Their results showed that including the assessment of consciousness as final step of the algorithm increases the sensitivity for patients in need of immediate lifesaving intervention by at least 5 per cent.

Ashour \& Kremer $^{4}$ present the triage algorithm that uses fuzzy analytic hierarchy process (FAHP) and multi-

Received : 27 March 2016, Revised : 29 April 2016

Accepted : 11 July 2016, Online published : 30 September 2016 attribute utility theory (MAUT) that rank patients based on chief complaint, age, gender, pain level, and vital signs. They compare the proposed algorithm and system with another one using discrete event simulation (DES). They are concluding that there is no strong statistical evidence that would suggest any improved performances of the proposed algorithm.

Scerbo ${ }^{5}$, et al. developed pre-hospital triage of trauma patients using the Random Forest algorithm. They conclude that the proposed algorithm could potentially be used to guide triage decisions despite the high negative predictive rate.

$\mathrm{Zhu}^{6}$, et al. and Saghafian ${ }^{7}$, et al. have investigated the possibility of application of Markov decision process models in hospital elective admissions and patient safety. They are proving that although misclassification of a complex patient is possible, slight variation from standard medical protocols could lead to relatively robust patient classification.

In this paper an IT architecture for collecting physiological data from all TMs over tactical military networks is proposed and proved, as a first stage in the process of defining an automated FRs' tool for estimating triage priorities.

\section{SYSTEM ARCHITECTURE FOR COLLECTING AND PROCESSING PHYSIOLOGICAL DATA}

The overall system architecture represents a data network, over which collected data packets can be transmitted from e-health sensors up to the main database servers in the cloud. The architecture has been developed to take the advantage 
of the existing military communication equipment on the battlefield as a standing point that needs to be upgraded.

A typical setup for military radio communications, on the lower tactical level, has been made over Ultra High Frequency (UHF) B band networks. Most of the military team radios operate in this UHF B range (250 MHz upto $500 \mathrm{MHz})^{8}$. This bandwidth allows transmitting of necessary data packets and voice calls simultaneously ${ }^{9}$. For our purpose, we have used this technology to transmit collected data packets from the TMs radio unit in FRs' radio unit and up to the units' cloud infrastructure.

As shown in Fig. 1, besides being part of the team, each member has a specific task to cover. It is always one person of the team who is designated to be a team leader (TL) and one person is designated to be an $\mathrm{FR}^{10}$. The goal of our project has been building a helpful tool for the FR and, therefore, all collected data must end up in the FRs tablet computer. At the same time, the collected data will be sent through TL communication equipment over to the cloud infrastructure for different monitoring purposes.

All collected data from e-health sensors are transferred over to the TMs radio unit, which in our project has acted as a gateway for transferring collected data into the units' tactical network. This connection can be wired or wireless which depends on radio unit specifications. The wired connection can be UDP or TCP/IP data stream in most of the today's tactical radios; however, wireless communication is more convenient and it is usually performed with Bluetooth Smart technology.

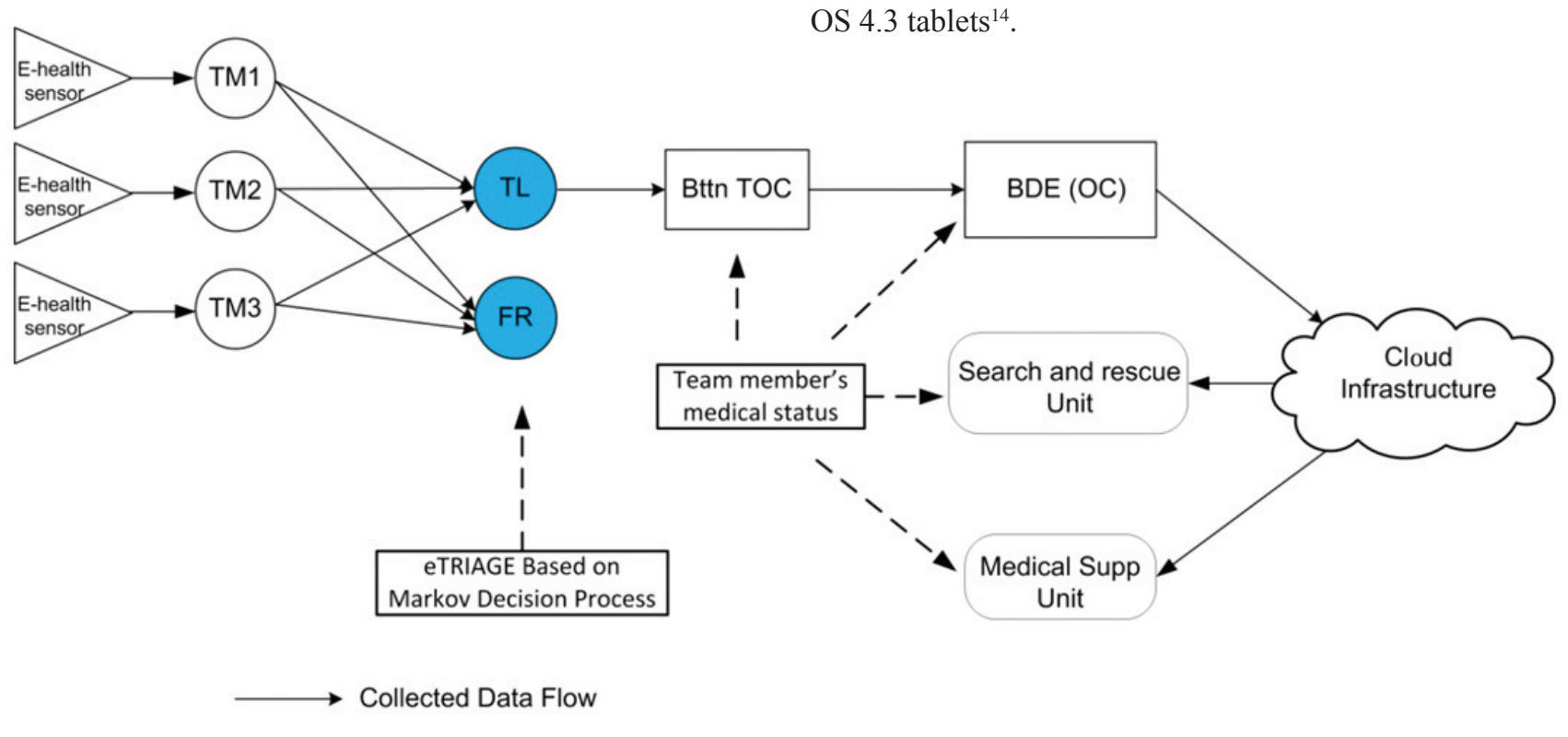

Figure 1. System architecture for real-time e-health monitoring.
By utilising the Bluetooth Smart/IEEE 802.15.6 technology, the new generation of e-health sensors have provided unique abilities in power consumption $(0.01 \mathrm{~W}$ to $0.05 \mathrm{~W}$ ) and data transfer (1 Mbps - $3 \mathrm{Mbps})^{11}$. These features are improving the longevity of the sensors and reliability in data transfer. This technology at the moment is a built-in feature in the new generations of portable radios ${ }^{12}$.

The true value of the project can be seen at the FRs' smart device unit. Collected data from all TMs is transmitted to this smart device where the physiological status of all TMs is estimated by processing the collected data about the respiratory rate, heart rate, blood pressure, and temperature. By custom developed application, the FR can monitor physiological status of all TMs. It can have information whether they are alive, tired, injured or dead. In addition, the application can categorise the victims by health risk factor (HRF) in the case of a multi-casualty incident.

Communication over the TL's radio is done only for transferring the collected data packets over to cloud network infrastructure. This leaves us with endless possibilities for data management and data usage by integrating the data on the different battle management and health monitoring systems.

\section{EXPERIMENTAL SETUP}

For the purpose of testing the main application, we have built a testing infrastructure using Harris Cor. Falcon III hardware. As shown in Fig. 2, the setup is made of sensors Arduino, two RF7800S UHF radios ${ }^{13}$ and two RF3590 Android OS 4.3 tablets $^{14}$.

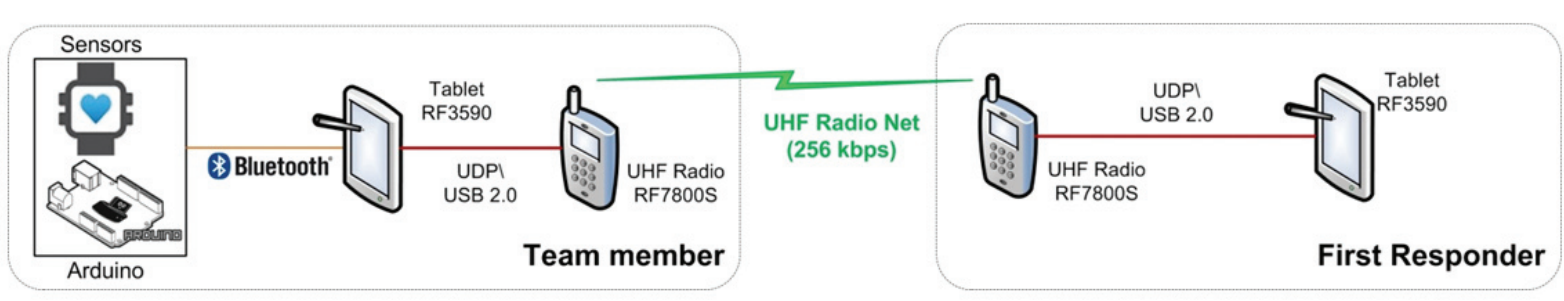

Figure 2. Laboratory setup for data transfer testing. 
Four different sensors were used to measure the vital parameters as follows: systolic blood pressure, heart rate, respiratory rate and temperature. The outputs of all these sensors were connected to the Arduino board through Sensor shield ${ }^{15}$. For transferring the data from the Arduino board to the Android tablets, we used $2.4 \mathrm{GHz}$ Bluetooth module and from the Android tablets to the UHF radios we used proprietary USB 2.0 interfaces connected by data cable. In real time scenario, this Bluetooth communication will be between the e-health sensors and TMs' radio unit.

A key part of the setup is the smart device on FRs' side. The performance and technical specifications were more than acceptable for our algorithm to operate without problems. With $7 "$ screen, this tablet provided enough space for visual data to be displayed even on a sunny day, or bright light.

Harris Falcon III RF7800S/SPR works on UHF frequency range from $350 \mathrm{MHz}$ upto $450 \mathrm{MHz}$. Enforcing Frequency-shift keying (FSK) and Gaussian frequency-shift keying (GFSK) modulation this radio has a capacity of 256 kbps wideband waveform transfer rate, over UHF radio communication ${ }^{12}$. This gives us a very good opportunity to simulate the data transfer between TMs to FR.

A setup like this is using many distinct components and requires the use of different types and protocols of communication. Starting from the input, sensors connected to Arduino was using a serial communication (also known as a UART). The data from the four sensors was then packed in a single frame using Arduino microcontroller and transmitted to Android tablet using Bluetooth. The frame structure is presented in the Table 1.

The first bit is ASCII character labeled as the start of text (STX). Then, data is following from each sensor separated with field separator (FS). After the last field separator, checksum was calculated and added at the end of the frame. Finally, the frame was closed with the end of the text (ETX). The purpose of the checksum is to confirm the correctness of the received data at the side of the tablets. The Checksum is computed on all characters, including all the $<\mathrm{FS}>$ characters, between STX and last FS. The checksum is calculated by the 8-bit binary addition of all included characters with the 8th or parity bit assumed to be zero. Carries beyond the 8th bit are lost. The 8-bit result is converted into two printable ASCII Hex characters, ranging from 00 to FF, which is then inserted into the data stream. The receiving device recalculates the checksum of the buffered message and compares it with the checksum it received. Any frame, which don't match the same checksum are considered as corrupted and not used in the following computation of the algorithm. The data type was an integer for all sensors.

Communication between the Android tablet and radio device is done with UDP over USB cable. This is high-speed communication and allows transmitting data over the TM smart device to FR tablet. The tablet has a built-in network adapter and drivers for communication over USB with a radio device (RF7800S). Both tablets were configured to run on the same subnet, but on different IP address and port number (Fig. 3).

Both client and server applications capable of exchanging the sensor data were developed. The client application, deployed on each TM tablet was running as a background service, which makes it more reliable. It was aimed at the real-time acquisition of the vital signs and their broadcasting (transmission) to FR, TL, as well as to cloud infrastructure. To accomplish real-time communication with multiple clients, we used multiplexer technique. The server application, deployed on FR and TL tablets, was composed of two parts, or to be more specific the background service and the GUI. Background service was used to listen for any received data and it was immediately processed and stored in the local database. However, the main goal of this service is to calculate the HRF by custom developed algorithm and to visualise it via the GUI (Fig. 4).

Algorithm we used to calculate the HRF was based on modified early warning score (MEWS) Table ${ }^{16}$ and Markov decision process for automating the medical emergency protocol (MDP-AMEP).

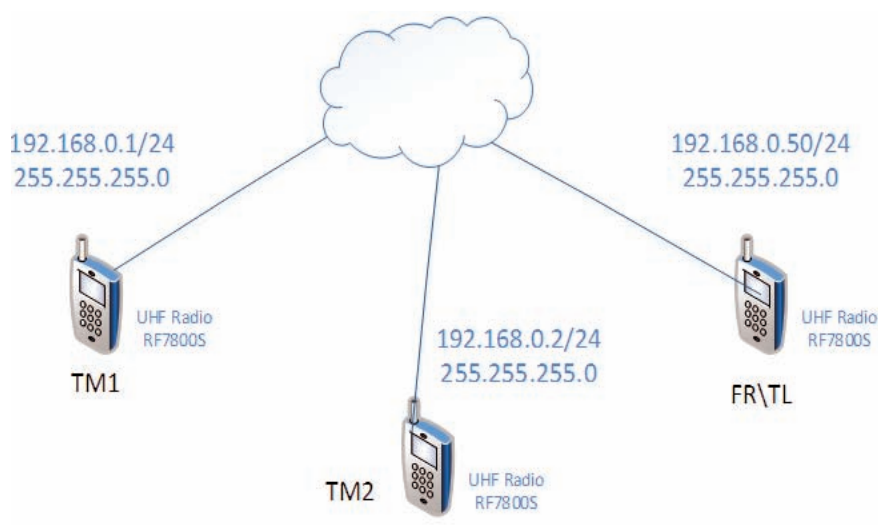

Figure 3. Interface parameters for data transfer in a radio environment.

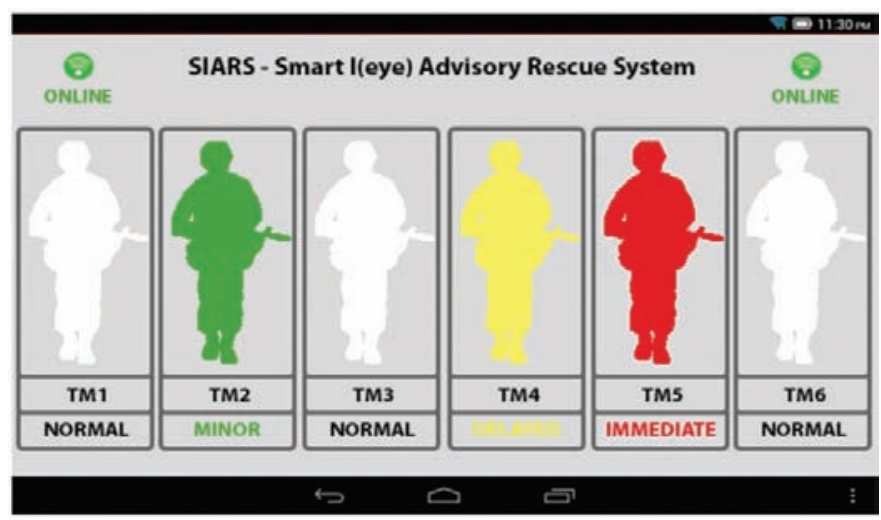

Figure 4. First responders' application interface.

Table 1. The frame of message format, data types, and size for each parameter

\begin{tabular}{ccccccccccc}
\hline STX & Heart Rate & FS & Resp. Rate & FS & Blood press. & FS & Temperature & FS & CKS & ETX \\
\hline & Int (3) & & $\operatorname{Int}(3)$ & & Int (3) & & Int (3) & & Char (2) & \\
\hline $\mathbf{1}$ & 8 & 1 & 8 & 1 & 8 & 1 & 8 & 1 & 2 & 1 \\
\hline
\end{tabular}


DEF. SCI. J., VOL. 66, NO. 5, SEPTEMBER 2016

Table 2. Test scenarios with medical vital signs and the color classification

\begin{tabular}{cccccccccc}
\hline Parameters & \multicolumn{4}{c}{ MDP Results (Part One) } & \multicolumn{3}{c}{ (AV) Part Two } \\
\hline Respiratory rate & 15 & 20 & 20 & 11 & 11 & 29 & 20 & 20 \\
Heart rate & 76 & 54 & 54 & 80 & 80 & 23 & 132 & 132 \\
Blood pressure & 132 & 140 & 140 & 99 & 99 & 28 & 140 & 140 \\
Temperature & 37 & 36.7 & 36.7 & 39.6 & 37.6 & 33 & 36.7 & 39.6 \\
AVPU score & 2 & 1 & 2 & 3 & 1 & 3 & 2 & 2 \\
Risk factor & Minor & Minor & Delayed & Delayed & Minor & Immediate & Immediate & Immediate \\
MEWS Score & 1 & 1 & 2 & 2 & 1 & 3 & 3 & 3 \\
\hline
\end{tabular}

\section{RESULTS AND DISCUSSION}

During the testing phase, we measured a delay in sending packages from TM to FRs' device to be in the range from $10 \mathrm{~ms}$ as a best case scenario, to $600 \mathrm{~ms}$ as a worst case scenario. The delay depended on the environment the radio device was in, and the network usage. Assuming this delay, we design our clientserver application to listen for data every 1s. This means every $1 \mathrm{~s}$, the server will receive one measurement from one TM. In such a situation where an injury occurs, determination of the health condition using these five sensors is much faster than without this equipment which can go up to $3 \mathrm{~h}-4 \mathrm{~h}$ or in some situations even more.

We used our equipment and environment to measure 8 different patients. The health condition of the patients was stable and we received the data shown in Table 2, part one. Because of the real data which didn't match any of the high-risk states, we inputted artificially values to represent the outcome of the algorithm in full range, part two.

Obtained results of our application, confirm that successful data transfer from e-health sensors to FRs' smart device can be established. This makes our proposed architecture acceptable for implementation in a real military environment where the physiological data is sent to the FR. Final determination of the HRF depends on the used algorithm for automated medical triage, which can be a subject for future research and development.

\section{CONCLUSIONS}

From testing, we can emphasise that successful physiological status monitoring can be implemented with current military communication assets. This can lead to improved medical triage and minimised death rate for engaging personnel. FR will have a real-time overview of his TMs physiological status and can give much faster and more relevant medical triage.

Furthermore, the collected data can have significant impact on the process of planning further units' missions. By database integration with present battle management systems, collected physiological data could improve the command and control capabilities of the engaged unit.

\section{REFERENCES}

1. Fitz Gerald, G.; Jelinek, G.; Scott D. \& Gerdtz M. Emergency department triage revisited. Postgraduate Medical J., 2010, 86(1018), 502-8.

doi:10.1136/emj.2009.077081
2. Ellebrecht, N. \& Kaufmann, S. Boosting efficiency through the use of IT? Int. J. Info. Syst. Crisis Response Management, 2014, 6(4), 1-18. doi:10.4018/ijiscram.2014100101

3. Horne, S.; Vassallo, J.; Read, J. \& Ball, S. UK triage-an improved tool for an evolving threat. Injury, 2013, 44(1), 23-8. doi:10.1016/j.injury.2011.10.005

4. Ashour, M. \& Kremer, O. A simulation analysis of the impact of FAHP-MAUT triage algorithm on the emergency department performance measures. Expert Syst. Appl., 2013, 40(1), 177-187. doi : 10.1016/j.eswa.2012.07.024

5. Scerbo, M.; Radhakrishnan, H.; Cotton, B.; Dua, A.; Del Junco, D.; Wade, C. \& Holcomb, J. B. Pre-hospital triage of trauma patients using the random forest computer algorithm. J. Surgical Res., 2014, 187(2), 371-376.

doi : 10.1016/j.jss.2013.06.037

6. Zhu, G.; Lizotte, D. \& Hoey, J. Scalable approximate policies for Markov decision process models of hospital elective admissions. Artificial Intel. Med., 2014, 61(1), 21-34. doi:10.1016/j.artmed.2014.04.001

7. Saghafian, S.; Hopp, W.J.; Van Oyen, M.P.; Desmond, J.S. \& Kronick, S.L. Complexity-augmented triage: A tool for improving patient safety and operational efficiency. Manufacturing Service Operations Manag., 2014, 16(3), 329-45. doi : 10.1287/msom.2014.0487

8. IEEE Terminology WG. 521-2002 - IEEE Standard Letter Designations for Radar-Frequency Bands, IEEE, 2002. doi : 10.1109/ieeestd.2003.94224

9. Cadman, P. Data transmission over VHF and UHF land mobile radio channels. Communications, Radar Signal Proc., 1983, 527 - 531. doi : 10.1049/ip-f-1.1983.0083

10. US Army. FM 3-21.8 The infantry rifle platoon and squad. Department of the Army, Washington, DC, 2007, 6-1.

11. Gomez, J. Overview and evaluation of bluetooth low energy: An emerging low-power wireless technology. Sensors, 2012, 11734-11753. doi : 10.3390/s120911734

12. Harris Corporation. Harris Corporation Introduces XG-25 portable radio for first responders. http://harris.com/view pressrelease.asp?act=lookup\&pr_id=3401. [Accessed 25 April 2016]

13. Fostea, D.; Barbu, A.; Arseni, S. \& Boiangiu, L. Testing state of the art radio communications equipments with regard to the legacy systems used in tactical environment. Scientific Bulletin Mircea cel Batran” Naval Academy, 2015, 18(2),112.

14. Agre, JR.; Gordon, KD. \& Vassiliou, M.S. Commercial 
technology at the tactical edge. Institute for defense analyses, Alexandria VA, 2013.

15. Khelil, A.; Shaikh, F.K.; Sheikh, A.A.; Felemban, E. \& Bojan, H. Digiaid: A wearable health platform for automated self-tagging in emergency cases. In Wireless Mobile Communication and Healthcare (Mobihealth), 2014 EAI 4th International Conference, IEEE, 2014. doi : 10.4108/icst.mobihealth.2014.257541

16. Subbe, C. P.; Kruger, M.; Rutherford, P. \& Gemmel, L. Validation of a modified early warning score in medical admissions. QJM, 2001, 94(10), 521-526.

doi : 10.1093/qjmed/94.10.521

\section{ACKNOWLEDGEMENT}

This work was supported by NATO Science for Peace and Security Programme, Project ISEG.EAP.SFPP 984753 - SIARS (Smart I (EYE) Advisory Rescue System). The authors are grateful for the technical support from the company NETRA Ltd. Skopje, Republic of Macedonia.

\section{CONTRIBUTORS}

Mr Goce Stevanoski is a Master student. He is experienced in planning and establishing military communication networks with different type of communication equipment. His field of interests are computer network design and cyber security. In the current study, his contribution: Conducted the experimental evaluation and data collection. The work included integration of the testing hardware and software infrastructure.

Mr Ivica Kocev has obtained his MSc in Faculty of computer science, University 'GoceDelcev' - Stip, Macedonia. Currently he is working in the field of software developing for bio sensors and its applications.

As an experienced software developer he built the server-side application, made the application GUI design and worked on the MDP-AMEP specification, implementation and development.
Dr Jugoslav Achkoski received his PhD (Computer Science) from the Ss. Cyril and Methodius University, Skopje, Macedonia in 2013. Currently working as an Assistant Professor in Military Academy 'General Mihailo Apostolski' - Skopje. His research interest includes: general theory of systems, distributed computers systems, computer architecture and organisation, machine learning algorithms. Currently he is working in the field of machine learning algorithms and bio sensors and its applications.

In the current study, his contribution: Associating indesigning the MDP-AMEP and conducting evaluation of results.

Dr Saso Koceski obtained his $\mathrm{PhD}$ in robotics and artificial intelligence in 2009 from the University of L'Aquila, Italy. Currently he is an associate professor at the Faculty of Computer Science, University 'GoceDelcev'-Stip, Macedonia and head of the Institute of Computer Science. His current research interests are focused in the field of artificial intelligence, robotics, bioengineering, HCI and medical imaging.

In the current study, his contribution: Idea and concept development, design of the system architecture and experimental setup.

Mr Boban Temelkovski, Skopje, Macedonia. He is a graduate engineer of Computer Science and System Engineering. His research interest includes: Theory of systems, electric drives and electromechanical systems, motors and drives, internet innovations, architecture of computers and operating systems, automatic control systems and embedded computer systems. Currently he is working in the field of bio sensors and its applications.

In the current study, his contribution to the project by developing the client side application and working on MDP-AMEP specification, implementation, and development. 